\title{
THE ITERATED WEAK HILBERT PROCEDURE
}

\author{
MARTIN DEFANT AND MARIUS JUNGE
}

(Communicated by William J. Davis)

\begin{abstract}
Applying Pisier's concept of weak properties to weak Hilbert spaces we obtain the so-called weak weak Hilbert spaces. Our main result is that the classes of weak Hilbert spaces and of weak weak Hilbert spaces coincide. On the other hand we show that a generalization for operators does not hold.
\end{abstract}

\section{INTRODUCTION}

In the following we introduce a general concept of how to produce weak ideals, in particular, those of weak weak Hilbert operators. For this let $(A, \alpha)$, $(B, \beta)$ be quasi-Banach ideals, $X, Y$ Banach spaces, and $T: X \rightarrow Y$ an operator. We say that $T \in Q(\alpha, \beta)$ [the quotient of $(A, \alpha)$ and $(B, \beta)$ ] if there is a constant $c \geq 0$ such that for all operators $u \in A\left(l_{2}, X\right)$

$$
\beta(T u) \leq c \alpha(u) \text {. }
$$

From this we deduce easily by trace duality that for all operators $u \in A\left(l_{2}, X\right)$, $v \in B^{*}\left(Y, l_{2}\right)$,

$$
\sum_{k \in \mathbb{N}} a_{k}(v T u) \leq c \alpha(u) \beta^{*}(v),
$$

where $a_{k}$ denotes the $k$ th approximation number and $\left(B^{*}, \beta^{*}\right)$ the conjugate ideal of $(B, \beta)$. For more precise definitions see the preliminaries below.

By Pisier's [Pi2] concept of weak properties we say that $T \in W(\alpha, \beta)$ (the weak ideal of $(A, \alpha)$ and $(B, \beta))$ if there is a constant $c \geq 0$ such that for all operators $u \in A\left(l_{2}, X\right), v \in B^{*}\left(Y, l_{2}\right)$,

$$
\sup _{k \in \mathbb{N}} k a_{k}(v T u) \leq c \alpha(u) \beta^{*}(v) .
$$

Observe that $(\mathrm{W})$ is obtained by replacing in $\left(\mathrm{Q}^{*}\right)$ the $l_{1}$-norm by the weaker $l_{1, \infty}$-norm. If we define $q(\alpha, \beta)(T)=\inf c, w(\alpha, \beta)(T)=\inf c$, where the infimum is taken over all $c$ such that $(\mathrm{Q}),(\mathrm{W})$ holds, respectively, then $(Q(\alpha, \beta), q(\alpha, \beta)),(W(\alpha, \beta), w(\alpha, \beta))$ are quasi-Banach ideals and

$$
Q(\alpha, \beta) \subset W(\alpha, \beta), \quad w(\alpha, \beta) \leq q(\alpha, \beta) .
$$

Received by the editors December 20, 1990.

1991 Mathematics Subject Classification. Primary 46B20, 47A30, 47A65.

Key words and phrases. Weak Hilbert spaces, weak weak Hilbert spaces. 
In this paper our aim is to apply this general scheme to the ideal norms $\alpha=\pi_{2}^{d}$ and $\beta=\pi_{2}$, where $\left(\Pi_{2}, \pi_{2}\right)$ denotes the Banach ideal of absolutely 2-summing operators and $\left(\Pi_{2}^{d}, \pi_{2}^{d}\right)$ the dual Banach ideal of $\left(\Pi_{2}, \pi_{2}\right)$ (i.e., $\left.\pi_{2}^{d}(u)=\pi_{2}\left(u^{*}\right)\right)$. Then by a well-known result of Kwapien [K] $T \in Q\left(\pi_{2}^{d}, \pi_{2}\right)$ iff $T$ is a Hilbert operator (operators, that factor through a Hilbert space). Applying (W) leads to Pisier's [Pil] definition of weak Hilbert operators, hence $T$ is a weak Hilbert operator if there is a constant $c \geq 0$ such that for all operators $u \in \Pi_{2}^{d}\left(l_{2}, X\right), v \in \Pi_{2}\left(Y, l_{2}\right)$,

$$
\sup _{k \in \mathbb{N}} k a_{k}(v T u) \leq c \pi_{2}\left(u^{*}\right) \pi_{2}(v)
$$

(recall that $\pi_{2}^{*}=\pi_{2}$ ). A result of Pisier [Pil] (see also [DJ]) says that $T$ is a weak Hilbert operator iff there is a constant $c \geq 0$ such that for all operators $u \in \Pi_{2}^{d}\left(l_{2}, X\right)$,

$$
\sup _{k \in \mathbb{N}} k^{1 / 2} a_{k}(T u) \leq c \pi_{2}\left(u^{*}\right)
$$

but this means that $T$ is a weak Hilbert operator iff $T \in Q\left(\pi_{2}^{d}, l_{2, \infty}^{a}\right)$, where $\left(\mathscr{L}_{2, \infty}^{a}, l_{2, \infty}^{a}\right)$ is the quasi-Banach ideal such that the quasi-ideal norm

$$
l_{2, \infty}^{a}(u)=\sup _{k \in \mathbb{N}} k^{1 / 2} a_{k}(u)<\infty .
$$

Hence we are in the situation to reapply (W) to $\alpha=\pi_{2}^{d}$ and $\beta=l_{2, \infty}^{a}$. This leads to the definition of the weak weak Hilbert operators.

$T$ is a weak weak Hilbert operator if there is a constant $c \geq 0$ such that for all operators $u \in \Pi_{2}^{d}\left(l_{2}, X\right), v \in\left(\mathscr{L}_{2, \infty}^{a}\right)^{*}\left(Y, l_{2}\right)$,

$$
\sup _{k \in \mathbb{N}} k a_{k}(v T u) \leq c \pi_{2}\left(u^{*}\right)\left(l_{2, \infty}^{a}\right)^{*}(v) .
$$

A Banach space $X$ is called weak Hilbert space, weak weak Hilbert space if $\mathrm{id}_{X}$ is a weak Hilbert operator, weak weak Hilbert operator, respectively. Our main results are the following:

1. Theorem. The classes of weak Hilbert spaces and weak weak Hilbert spaces coincide.

2. Theorem. There is a weak weak Hilbert operator, which is not a weak Hilbert operator.

\section{PRELIMINARIES}

We use standard Banach space notations. In particular, we have for all Banach spaces $X$ and subspaces $E \subset X: i_{E}: E \rightarrow X, x \mapsto x$. The Lorentz sequence spaces $\left(l_{p, q},\|\circ\|_{p, q}\right),\left(l_{p, q}^{n},\|\circ\|_{p, q}\right), n \in \mathbb{N}, 0<p, q \leq \infty$, are defined in the usual way. Standard references on $s$-numbers and operator ideals are the monographs of Pietsch [P1, P2]. The ideal of all linear bounded, finite-dimensional operators are denoted by $\mathscr{L}, \mathscr{F}$, respectively.

Let $(A, \alpha)$ be a quasi-Banach ideal. The component $A^{*}(X, Y)$ of the conjugate ideal $\left(A^{*}, \alpha^{*}\right)$ is the class of all operators $T \in \mathscr{L}(X, Y)$ such that

$$
\alpha^{*}(T)=\sup \{|\operatorname{tr} T S| \mid S \in \mathscr{F}(Y, X), \alpha(S) \leq 1\}<\infty .
$$


The component $A^{d}(X, Y)$ of the dual ideal $\left(A^{d}, \alpha^{d}\right)$ is the class of all operators $T \in \mathscr{L}(X, Y)$ such that $T^{*} \in A\left(Y^{*}, X^{*}\right)$. We set

$$
\alpha^{d}(T)=\alpha\left(T^{*}\right) .
$$

We call a quasi-Banach ideal $(A, \alpha)$ injective if we have for all operators $T \in \mathscr{L}(X, Y)$ and isometries $I \in \mathscr{L}(X, Z)$ with $I T \in A(X, Z)$ that $T \in$ $A(X, Y)$ and $\alpha(T)=\alpha(I T)$.

Note that for all two quasi-Banach ideals $(A, \alpha),(B, \beta)$ the inclusion $A \subset$ $B$ implies $\beta \leq c \alpha$ for some constant $c \geq 0$.

Next we recall the usual notation of some $s$-numbers of an operator $T \in$ $\mathscr{L}(X, Y)$.

$$
\begin{aligned}
a_{n}(T)= & \inf \{\|T-S\| \mid S \in \mathscr{L}(X, Y), \operatorname{rank}(S)<n\} \\
& \text { the } n \text {th approximation number, } \\
x_{n}(T)= & \sup \left\{a_{n}(T u) \mid u \in \mathscr{L}\left(l_{2}, X\right),\|u\| \leq 1\right\} \\
& \text { the } n \text {th Weyl number, } \\
h_{n}(T)= & \sup \left\{a_{n}(v T u) \mid u \in \mathscr{L}\left(l_{2}, X\right),\|u\| \leq 1, v \in \mathscr{L}\left(Y, l_{2}\right),\|v\| \leq 1\right\} \\
& \text { the } n \text {th Hilbert number. }
\end{aligned}
$$

If $X$ is a Hilbert space then $a_{n}(T)=x_{n}(T)$ and $a_{n}(T)=a_{n}\left(T^{*}\right)$. The Weyl numbers are injective, i.e., if $S \in \mathscr{L}(Y, Z)$ is an isometrie then $x_{n}(T)=$ $x_{n}(I T)$. Furthermore they are multiplicative, i.e., for all operators $S \in \mathscr{L}(Y, Z)$ and $n, m \in \mathbb{N}, x_{m+n-1}(S T) \leq x_{n}(S) x_{m}(T)$. Recall that the Hilbert numbers are completely symmetric, i.e., $h_{n}(T)=h_{n}\left(T^{*}\right)$.

Now let $s$ be any $s$-number. The component $\mathscr{L}_{p, q}^{s}(X, Y)$ of the quasiBanach ideal $\left(\mathscr{L}_{p, q}^{s}, l_{p, q}^{s}\right)$ is the class of all operators $T \in \mathscr{L}(X, Y)$ such that

$$
l_{p, q}^{s}(T)=\left\|\left(s_{k}(T)\right)_{k}\right\|_{p, q}<\infty .
$$

Since the Weyl numbers are injective, it is obvious that the Weyl ideal $\left(\mathscr{L}_{p, q}^{x}\right.$, $\left.l_{p, q}^{x}\right)$ is injective.

An operator is called a Hilbert operator $\left(T \in \Gamma_{2}(X, Y)\right)$ if there is a Hilbert space $H$ and operators $S \in \mathscr{L}(X, H), R \in \mathscr{L}(H, Y)$ such that $T=R S$. We denote by $\gamma_{2}(T)=\inf \|R\|\|S\|$, where the infimum is taken over all such factorizations through a Hilbert space.

An operator is said to be absolutely 2-summing $\left(T \in \Pi_{2}(X, Y)\right)$ if there is a constant $c \geq 0$ such that for all $n \in \mathbb{N},\left(x_{k}\right)_{k=1}^{n} \subset X$,

$$
\left(\sum_{k=1}^{n}\left\|T x_{k}\right\|^{2}\right)^{1 / 2} \leq c \sup _{x^{*} \in B_{X^{*}}}\left(\sum_{k=1}^{n}\left|\left\langle x_{k}, x^{*}\right\rangle\right|^{2}\right)^{1 / 2} .
$$

We let $\pi_{2}(T)=\inf c$, where the infimum is taken over all $c$ such that the inequality holds. Recall that $\Pi_{2}=\Pi_{2}^{*}$ with equal norms [P2].

$\left(\Gamma_{2}, \gamma_{2}\right)$ and $\left(\Pi_{2}, \pi_{2}\right)$ are Banach ideals [P2].

At the end of the preliminaries we want to mention those known results that we use essentially in this paper.

(1) [P1] Let $H$ be a Hilbert space, $T \in \mathscr{L}(H, Y)$, and $n \in \mathbb{N}$. If $a_{n}(T)>0$, then for every $\varepsilon>0$ there is an orthogonal family $\left(x_{k}\right)_{k=1}^{n} \subset H$ such that $a_{k}(T) \leq(1+\varepsilon)\left\|T x_{k}\right\|$ for all $k=1, \ldots, n$. 
(2) [P2] Let $T \in \mathscr{L}(X, Y), n \in \mathbb{N}$, and $\varepsilon>0$. Then there are operators $S \in \mathscr{L}\left(l_{2}^{n}, X\right), R \in \mathscr{L}\left(Y, l_{2}^{n}\right)$ such that $\|R\| \leq 1,\|S\| \leq(1+\varepsilon) / h_{n}(T)$, and $\mathrm{id}_{l_{2}^{n}}=R T S$.

(3) [Jo, Pi2, T-J] Let $n \in \mathbb{N}$ and $E$ be a Banach space with $\operatorname{dim} E=n$. Then there is an invertible operator $u \in \mathscr{L}\left(l_{2}^{n}, E\right)$ such that $\|u\|=1$ and $\pi_{2}\left(u^{-1}\right)=n^{1 / 2}$.

(4) [P1] $\Pi_{2} \subset \mathscr{L}_{2, \infty}^{x}$ and $l_{2, \infty}^{x} \leq \pi_{2}$.

(5) [DJ] Let $(A, \alpha)$ be an injective Banach ideal and $(B, \beta)$ an injective quasi-Banach ideal. Then $W\left(a^{* d}, \beta\right)=W\left(\beta^{* d}, \alpha\right)^{d}$.

(6) [DJ] Let $(A, \alpha),(C, \gamma)$ be quasi-Banach ideals and $(f(n))_{n}$ be a positive sequence such that $c=\sup _{n \in \mathbb{N}}\left(\prod_{k=1}^{n} f(k) / f(n)\right)^{1 / n}<\infty$. Then we have for all $T \in \mathscr{L}(X, Y)$ the implication (i) $\rightarrow$ (ii) and the estimate $M_{2}(T) \leq M_{1}(T)$.

(i) There is a constant $M_{1}(T) \geq 0$ such that for all $u \in A\left(\ell_{2}, X\right), v \in$ $\Gamma\left(Y, l_{2}\right), \sup _{k \in \mathbb{N}} f(k) a_{k}(v T u) \leq M_{1}(T) \alpha(u) \gamma(v)$.

(ii) There is a constant $M(T) \geq 0$ such that for all $u \in A(l, X)$,

$$
\sup _{k \in \mathbb{N}} \frac{f(k)}{\gamma\left(\mathrm{id}: l_{\infty}^{k} \rightarrow l_{2}^{k}\right)} a_{k}(T u) \leq M_{2}(T) \alpha(u)
$$

(7) $[\mathrm{K}] \Gamma_{2}=Q\left(\pi_{2}^{d}, \pi_{2}\right)$ with equal norms.

(8) [Pi2] $A$ Banach space $X$ is a weak Hilbert space (id ${ }_{X} \in W\left(\pi_{2}^{d}, \pi_{2}\right)$ ) iff there is a constant $c \geq 0$ and a number $0<\delta \leq 1$ such that for all $n \in \mathbb{N}$ with $\delta n \geq 1$ and subspaces $E \subset X$ with $\operatorname{dim} E=n$ there is a subspace $F \subset E$ with $\operatorname{dim} F=[\delta n]$ and a projection $P_{F} \in \mathscr{L}(X, F)$ onto $F$ with $\gamma_{2}\left(P_{F}\right) \leq c$.

To prove Theorem 1 we start with

3. Proposition. Let $X$ be a Banach space and $c \geq 0$ a constant such that for all operators $u \in\left(\mathscr{L}_{2, \infty}^{a}\right)^{* d}\left(l_{2}, X\right), v \in \Pi_{2}\left(X, l_{2}\right)$,

$$
\sup _{k \in \mathbb{N}} k a_{k}(v u) \leq c\left(l_{2, \infty}^{a}\right)^{*}\left(u^{*}\right) \pi_{2}(v) .
$$

Then $X$ is a weak Hilbert space.

Proof. Since $\pi_{2}\left(\mathrm{id}: l_{\infty}^{k} \rightarrow l_{2}^{k}\right)=k^{1 / 2}$, we deduce from the assumption, (6), and the multiplicity of the Weyl numbers that for all $w \in\left(l_{2, \infty}^{a}\right)^{* d}\left(l_{2}, X\right)$,

$$
\begin{aligned}
l_{2, \infty}^{a}(w) & =\sup _{k \in \mathbb{N}} k^{1 / 2} a_{k}(w) \leq e c\left(l_{2, \infty}^{a}\right)^{*}\left(w^{*}\right) \\
& \leq e c \sup \left\{\sum_{k \in \mathbb{N}} a_{k}\left(w^{*} u\right) \mid u \in \mathscr{F}\left(l_{2}, X^{*}\right), l_{2, \infty}^{a}(u) \leq 1\right\} \\
& \leq 2 e c \sum_{k \in \mathbb{N}} \frac{x_{k}\left(w^{*}\right)}{k^{1 / 2}}=2 e c \sum_{k \in \mathbb{N}} \frac{h_{k}(w)}{k^{1 / 2}} .
\end{aligned}
$$

Now choose $0<\delta \leq 1$ such that for all $n \in \mathbb{N}$,

$$
\sum_{k=1}^{[\delta n]} \frac{1}{k^{1 / 2}} \leq \frac{1}{8 e c} n^{1 / 2}
$$


$\left(\sum_{k=1}^{0} 1 / k^{1 / 2}=0\right)$. Fix $n \in \mathbb{N}$ with $\delta n \geq 1$ and a subspace $E \subset X$ with $\operatorname{dim} E=[\delta n] . \quad$ By (3) there is an invertible operator $u \in \mathscr{L}\left(l_{2}^{n}, E\right)$ with $\|u\|=1$ and $\pi_{2}\left(u^{-1}\right) \leq n^{1 / 2}$. Hence we obtain, with $m=(n+1) / 2 \geq n / 2$, the multiplicity and the injectivity of the Weyl numbers and, by (4),

$$
\begin{aligned}
n^{1 / 2} & \leq \sqrt{2} m^{1 / 2} a_{n}\left(\operatorname{id}_{l_{2}^{n}}\right) \leq \sqrt{2} m^{1 / 2} x_{m}(u) x_{m}\left(u^{-1}\right) \\
& \leq \sqrt{2} l_{2, \infty}^{x}(u) \frac{\pi_{2}\left(u^{-1}\right)}{m^{1 / 2}} \leq \sqrt{2} l_{2, \infty}^{x}(u)\left(\frac{n}{m}\right)^{1 / 2} \\
& \leq 2 l_{2, \infty}^{x}(u) \leq 2 l_{2, \infty}^{a}\left(i_{E} u\right) \leq 4 e c l_{2,1}^{h}\left(i_{E} u\right) \\
& \leq 4 e c\left(\sum_{k=1}^{[\delta n]} \frac{h_{k}\left(i_{E} u\right)}{k^{1 / 2}}+\sum_{k=[\delta n]}^{n} \frac{h_{k}\left(i_{E} u\right)}{k^{1 / 2}}\right) \\
& \leq 4 e c\left(\left\|i_{E} u\right\| \frac{1}{8 e c} n^{1 / 2}+\|u\| h_{[\delta n]}\left(i_{E} u\right) 2 n^{1 / 2}\right) \\
& \leq \frac{1}{2} n^{1 / 2}+8 e c h_{[\delta n]}\left(i_{E}\right) n^{1 / 2} ;
\end{aligned}
$$

but this implies $h_{[\delta n]}\left(i_{E}\right) \geq 1 / 16 e c$.

Let $\varepsilon>0$. Then by (2) there are operators $R \in \mathscr{L}\left(X, l_{2}^{[\delta n]}\right)$ and $S \in$ $\mathscr{L}\left(l_{2}^{[\delta n]}, X\right)$ such that $\|R\| \leq 1,\|S\| \leq(1+\varepsilon) / h_{[\delta n]}\left(i_{E}\right)$, and $R i_{E} S=\mathrm{id}_{l_{2}^{[\delta n]}}$. Then we define $F=S\left(l_{2}^{[\delta n]}\right) \subset E, \widetilde{S}: l_{2}^{[\delta n]} \rightarrow F, x \mapsto S(x) \in \mathscr{L}\left(l_{2}^{[\delta n]}, F\right)$, and $P_{F}=\tilde{S} R \in \mathscr{L}(X, F)$. Hence $\operatorname{dim} F=[\delta n], P_{F}$ is a projection onto $F$, and

$$
\gamma_{2}\left(P_{F}\right) \leq\|R\|\|\widetilde{S}\| \leq \frac{(1+\varepsilon)}{h_{[\delta n]}\left(i_{E}\right)} \leq(1+\varepsilon) 16 e c .
$$

Therefore (8) yields our assertion.

Now we are able to prove Theorem 1 .

Proof of Theorem 1. Assume that $X$ is a weak weak Hilbert space. This means $\operatorname{id}_{X} \in W\left(\pi_{2}^{d}, l_{2, \infty}^{a}\right)=W\left(\pi_{2}^{* d}, l_{2, \infty}^{x}\right)$. Since $\left(\Pi_{2}, \pi_{2}\right)$ is an injective Banach ideal and $\left(\mathscr{L}_{2, \infty}^{x}, l_{2, \infty}^{x}\right)$ is an injective quasi-Banach ideal. (5) implies that $\operatorname{id}_{X} \in\left(W\left(\left(l_{2, \infty}^{x}\right)^{* d}, \pi_{2}\right)^{d}\right.$; hence we deduce from Proposition 3 that $X^{*}$ is a weak Hilbert space. Then by Pisier [Pil] (or again (5)) it is well known that $X$ is a weak Hilbert space.

Remark. In fact, we proved that the assumption of Proposition 3 characterizes weak Hilbert space.

To prove Theorem 2 we need the following.

4. Lemma. Let $X$ be an Banach space and $v \in\left(\mathscr{L}_{2, \infty}^{a}\right)^{*}\left(X, l_{2}\right)$. Then

$$
\sum_{k \in \mathbb{N}} \frac{a_{k}(v)}{k} \leq \sqrt{2}\left(l_{2, \infty}^{a}\right)^{*}(v) .
$$

Proof. Let $n \in \mathbb{N}$ such that $a_{n}(v)=a_{n}\left(v^{*}\right)>0$. Then by (1) for $\varepsilon>0$ we find a complete orthonormal system $\left(e_{k}\right)_{k \in \mathbb{N}} \subset l_{2}$ and a sequence $\left(x_{k}\right)_{k=1}^{n} \subset B_{X}$ such that for all $k=1, \ldots, n$,

$$
a_{k}(v)=a_{k}\left(v^{*}\right) \leq(1+\varepsilon)\left\|v^{*}\left(e_{k}\right)\right\| \leq(1+\varepsilon)^{2}\left\langle x_{k}, v^{*}\left(e_{k}\right)\right\rangle .
$$


Let us define $u=\sum_{k=1}^{n} e_{k} \otimes x_{k} / k \in \mathscr{L}\left(l_{2}, X\right)$ and let $P_{k} \in \mathscr{L}\left(l_{2}, l_{2}\right)$ be a projection onto $\operatorname{span}\left\{e_{1}\right\}_{i=1}^{k-1}$ for $k=1, \ldots, n \quad\left(\operatorname{span}\left\{e_{i}\right\}_{i=1}^{0}=\{0\}\right)$. Then we have

$$
\begin{aligned}
a_{k}(u) & \leq\left\|u-u P_{k}\right\|=\left\|u_{\mid \operatorname{span}\left\{e_{i}\right\}_{i=k}^{\infty}}\right\|=\sup \left\{\left.\left\|\sum_{i=k}^{\infty} \alpha_{i} e_{i}\right\|\left|\sum_{i=k}^{\infty}\right| \alpha_{i}\right|^{2} \leq 1\right\} \\
& \leq\left(\sum_{i=k}^{\infty} \frac{\left\|x_{i}\right\|^{2}}{i^{2}}\right)^{1 / 2} \leq \sqrt{2} \frac{1}{k^{2}} .
\end{aligned}
$$

Hence this implies

$$
\begin{aligned}
\sum_{k=1}^{n} \frac{a_{k}(v)}{k} & \leq(1+\varepsilon)^{2} \sum_{k=1}^{n} \frac{1}{k}\left\langle x_{k}, v^{*}\left(e_{k}\right)\right\rangle \\
& \leq(1+\varepsilon)^{2} \sum_{k=1}^{n}\left\langle u\left(e_{k}\right), v^{*}\left(e_{k}\right)\right\rangle=(1+\varepsilon)^{2} \operatorname{tr} v u \\
& \leq(1+\varepsilon)^{2} l_{2, \infty}^{a}(u)\left(l_{2, \infty}^{a}\right)^{*}(v) \\
& =(1+\varepsilon)^{2} \sup _{k=1, \ldots, n} k^{1 / 2} a_{k}(u)\left(l_{2, \infty}^{a}\right)^{*}(v) \\
& \leq \sqrt{2}(1+\varepsilon)^{2}\left(l_{2, \infty}^{a}\right)^{*}(v) .
\end{aligned}
$$

Since $n \in \mathbb{N}$ and $\varepsilon>0$ are arbitrary, the assertion is proved.

5. Proposition. For every $n \in \mathbb{N}$ and every operator $T \in \mathscr{L}$ with $\operatorname{rank}(T)=n$ we have

$$
w\left(\pi_{2}^{d}, l_{2, \infty}^{a}\right)(T) \leq 8 \frac{n^{1 / 2}}{1+\ln n}\|T\| .
$$

Proof. Let $u \in \Pi_{2}^{d}\left(l_{2}, X\right)$ and $v \in\left(\mathscr{L}_{2, \infty}^{a}\right)^{*}\left(Y, l_{2}\right)$. Then the multiplicity of the Weyl numbers, (4), and Lemma 4 imply

$$
\begin{aligned}
\sup _{k \in \mathbb{N}} k a_{k}(v T u) & =\sup _{k \in \mathbb{N}} k x_{k}\left(u^{*} T^{*} v^{*}\right) \leq 2 l_{2, \infty}^{x}\left(u^{*}\right) l_{2, \infty}^{x}\left(T^{*} v^{*}\right) \\
& \leq 2 \pi_{2}\left(u^{*}\right) \sup _{k=1, \ldots, n} k^{1 / 2} x_{k}\left(T^{*} v^{*}\right) \\
& \leq 2 \pi_{2}\left(u^{*}\right)\|T\| \sup _{k=1, \ldots, n} \frac{k^{1 / 2}}{1+\ln k}(1+\ln k) a_{k}(v) \\
& \leq \frac{4}{\sqrt{e}} \frac{n^{1 / 2}}{1+\ln n}\|T\| \pi_{2}\left(u^{*}\right) \sup _{k=1, \ldots, n}(1+\ln k) a_{k}(v) \\
& \leq \frac{8}{\sqrt{e}} \frac{n^{1 / 2}}{1+\ln n}\|T\| \pi_{2}\left(u^{*}\right) \sum_{k \in \mathbb{N}} \frac{a_{k}(v)}{k} \\
& \leq 8 \frac{n^{1 / 2}}{1+\ln n}\|T\| \pi_{2}\left(u^{*}\right)\left(l_{2, \infty}^{a}\right)^{*}(v) .
\end{aligned}
$$

Hence the definition of the weak weak Hilbert norm completes the proof.

An immediate consequence is 
Proof of Theorem 2. Let $i_{2, \infty}^{n}: l_{2}^{n} \rightarrow l_{\infty}^{n}$ and $i_{\infty, 2}^{n}: l_{\infty}^{n} \rightarrow l_{2}^{n}$ for $n \in \mathbb{N}$ be the identity maps. Since $\pi_{2}^{d}\left(i_{2, \infty}^{n}\right)=1$ and $\pi_{2}\left(i_{\infty, 2}^{n}\right)=n^{1 / 2}$, we have

$$
n^{1 / 2} \leq w\left(\pi_{2}^{d}, \pi_{2}\right)\left(\operatorname{id}_{l_{\infty}^{n}}\right) \quad\left(\leq \gamma_{2}\left(\operatorname{id}_{l_{\infty}^{n}}\right)=n^{1 / 2}\right) .
$$

Now suppose that the quasi-Banach ideals of weak Hilbert operators and of weak weak Hilbert operators coincide. Hence there is a constant $c \geq 0$ such that

$$
n^{1 / 2} \leq w\left(\pi_{2}^{d}, \pi_{2}\right)\left(\operatorname{id}_{l_{\infty}^{n}}\right) \leq c w\left(\pi_{2}^{d}, l_{2, \infty}^{a}\right)\left(\operatorname{id}_{l_{\infty}^{n}}\right) \leq 8 c \frac{n^{1 / 2}}{1+\ln n} .
$$

But this is a contradiction and, therefore, the assertion is proved.

Remark. We are able to prove the following result for diagonal operators $D_{\sigma}$ : $l_{\infty} \rightarrow l_{\infty},\left(x_{k}\right)_{k \in \mathbb{N}} \mapsto\left(\sigma_{k} x_{k}\right)_{k \in \mathbb{N}}$

$$
D_{\sigma} \text { is a weak weak Hilbert operator iff } \sup _{k \in \mathbb{N}} \frac{k^{1 / 2}}{1+\ln k} \sigma_{k}^{*}<\infty \text {, }
$$

where $\left(\sigma_{k}^{*}\right)_{k \in \mathbb{N}}$ denotes the nonincreasing rearrangement of $\sigma$. On the other hand,

$$
D_{\sigma} \text { is a weak Hilbert operator iff } \sup _{k \in \mathbb{N}} k^{1 / 2} \sigma_{k}^{*}<\infty .
$$

Remark. Note that Theorem 2 implies that on weak Hilbert spaces the dependence of the weak Hilbert norm $w\left(\pi_{2}^{d}, \pi_{2}\right)$ and of the weak weak Hilbert norm $w\left(\pi_{2}^{d}, l_{2, \infty}^{a}\right)$ cannot be linear.

\section{REFERENCES}

[DJ] M. Defant and M. Junge, On weak $(r, 2)$-summing operators and weak Hilbert spaces, Studia Math. 46 (1990), 203-217.

[Jo] F. John, Extremum problems with inequalities as subsidiary conditions, Courant Anniversay Volume, Interscience, New York, 1948, pp. 187-204.

[K] S. Kwapien, A linear topological characterization of inner product spaces, Studia Math. 38 (1970), 277-278.

[P1] A. Pietsch, Eigenvalues and s-numbers, Geest \& Portig, Leipzig, 1987, and Cambridge Univ. Press, London and New York, 1987.

[P2] _ Operator ideals, Deutscher Verlag Wiss., Berlin, 1978, and North-Holland, Amsterdam, New York, and Oxford, 1980.

[Pil] G. Pisier, Weak Hilbert spaces, Proc. London Math. Soc. (3) 56 (1988), 547-579.

[Pi2] _ _ Volume of convex bodies and Banach space geometry, Cambridge Univ. Press, 1989.

[T-J] N. Tomczak-Jaegermann, Banach Mazur distances and finite-dimensional operator ideals, Harlow, Longman, and Wiley, New York, 1988.

Mathematisches Seminar, Universiät Kiel, Olshausenstrasse 40-60, D-2300 Kiel 1, GERMANY

E-mail address, Marius Junge: NMS006@rz.uni-kiel.dbp.de 